\title{
A NATURAL PARTIAL ORDER FOR SEMIGROUPS
}

\author{
H. MITSCH
}

Dedicated to E. A. Behrens on the occasion of his 70 th birthday

\begin{abstract}
A partial order on a semigroup $(S, \cdot)$ is called natural if it is defined by means of the multiplication of $S$. It is shown that for any semigroup $(S, \cdot)$ the relation $a \leq b$ iff $a=x b=b y, x a=a$ for some $x, y \in S^{1}$, is a partial order. It coincides with the well-known natural partial order for regular semigroups defined by Hartwig [4] and Nambooripad [10]. Similar relations derived from the natural partial order on the regular semigroup $\left(T_{X}, \circ\right)$ of all maps on the set $X$ are investigated.
\end{abstract}

1. Introduction. For any semigroup $(S, \cdot)$ the set $E_{S}$ of all idempotents is partially ordered by

$$
e \leq f \quad \text { iff } \quad e=e f=f e \quad\left(e, f \in E_{S}\right) .
$$

Since idempotents give a good deal of information on the semigroup, this partial order can be used successfully in the study of $(S, \cdot)$ as a whole. There have been numerous attempts to extend this ordering from $E_{S}$ to all of $S$ defining it by means of the multiplication of $S$ and postulating that its restriction to $E_{S}$ coincides with the partial order (1) (see Burgess and Raphael [2], Hartwig [4], Lawson [8], McAlister [9] and Nambooripad [10]). The reason for the interest in such natural orders lies in the obvious fact that such an ordering can provide additional information on a given semigroup since it reflects its multiplication in a particular way.

An important class of semigroups for which such a natural partial order was found is that of inverse semigroups. It was defined by V. Vagner in 1952 as follows:

$$
a \leq b \quad \text { iff } a=e b \text { for some } e \in E_{S} .
$$

Compatible on both sides with multiplication (i.e. $a \leq b$ implies $a c \leq b c$ and $c a \leq c b$ for all $c \in S$ ) it proved very useful in the theory of inverse semigroups. It took about 30 years before this order relation was generalized to the class of regular semigroups. This natural partial order was found by R. Hartwig [4] and K. Nambooripad [10] in 1980, independently. The most commonly used definition is the following:

$$
a \leq b \quad \text { iff } \quad a=e b=b f \quad \text { for some } e, f \in E_{S} .
$$

For $(S, \cdot)$ an inverse semigroup this relation is the partial order $(2)$ above. For $(S, \cdot)$ regular it is no longer compatible with multiplication on either side. Those regular semigroups $S$ for which $a \leq b$ implies $a c \leq b c$ and $c a \leq c b$ for all $c \in S$, respectively, were characterized by Blyth and Gomes [1], and for which both implications hold by K. Nambooripad [10].

Several equivalent definitions of this natural partial order on a regular semigroup are known. For completeness we list them all adding a new one (Lemma 1(x)).

Received by the editors April 29, 1985.

1980 Mathematics Subject Classification. Primary 20M99; Secondary 06F05. 
Since the latter will be used in the sequel, we provide a short proof. This will solve the problem of finding a natural partial order on any semigroup which for regular semigroups coincide with the known relation (3) above. In the following lemma condition (iv) is the definition given by R. Hartwig [4] and condition (vii) that of K. Nambooripad [10]; conditions (ii), (iii) are due to G. Gomes [3], and (v), (vi) to J. Hickey [5].

LEMMA 1. For a regular semigroup $(S, \cdot)$ the following are equivalent:

(i) $a=e b=b f$ for some $e, f \in E_{S}$,

(ii) $a=a a^{\prime} b=b a^{\prime \prime} a$ for some $a^{\prime}, a^{\prime \prime} \in V(a)=\{x \in S \mid a=a x a, x=x a x\}$,

(iii) $a=a a^{0} b=b a^{0} a$ for some $a^{0} \in V(a)$,

(iv) $a^{\prime} a=a^{\prime} b$ and $a a^{\prime}=b a^{\prime}$ for some $a^{\prime} \in V(a)$,

(v) $a=a b^{*} b=b b^{*} a, a=a b^{*} a$ for some $b^{*} \in V(b)$,

(vi) $a=a x b=b x a, a=a x a, b=b x b$ for some $x \in S$,

(vii) $a=e b$ for some idempotent $e \in R_{a}$ and $a S \subseteq b S$,

(viii) for every idempotent $f \in R_{b}$ there is an idempotent $e \in R_{a}$ with $e \leq f$ and $a=e b$,

(ix) $a=a b^{\prime} a$ for some $b^{\prime} \in V(b), a S \subseteq b S$ and $S a \subseteq S b$,

(x) $a=x b=b y, x a=a$ for some $x, y \in S$,

(xi) $a=e b=b x$ for some $e \in E_{S}, x \in S$.

PROOF. The equivalence of (i)-(iii) was proved by G. Gomes [3].

(iii) $\Rightarrow$ (iv) $a=a a^{0} b$ implies $a^{0} a=a^{0} \cdot a a^{0} b=a^{0} b$, and $a=b a^{0} a$ implies $a a^{0}=b a^{0} a \cdot a^{0}=b a^{0}$ since $a^{0} \in V(a)$.

(iv) $\Rightarrow$ (v) $a^{\prime} a=a^{\prime} b$ implies $a=a a^{\prime} b$, and $a a^{\prime}=b a^{\prime}$ implies $a=b a^{\prime} a$; hence, for $b^{*} \in V(b)$ we get

$$
a b^{*} a=a a^{\prime} b \cdot b^{*} \cdot b a^{\prime} a=a a^{\prime} b a^{\prime} a=a a^{\prime} b \cdot a^{\prime} a=a \cdot a^{\prime} a=a ;
$$

furthermore,

$$
a b^{*} b=a a^{\prime} b \cdot b^{*} b=a a^{\prime} b=a \text { and } \quad b b^{*} a=b b^{*} \cdot b a^{\prime} a=b a^{\prime} a=a .
$$

The equivalence of $(\mathrm{v})$ and (vi) was proved by J. Hickey [5].

(vi) $\Rightarrow$ (vii) From $a=a x a$ it follows that $a x \in E_{S}$, thus $a=a x b=e b$ with $e=a x \in R_{a}$; also, $a=b x a \in b S$ implies that $a S \subseteq b S$.

The equivalence of (vii)-(ix) was proved by K. Nambooripad [10].

(ix) $\Rightarrow(\mathrm{x})$ Since $(S, \cdot)$ is regular, $a S \subseteq b S$ and $S a \subseteq S b$ imply that $a=x b=b y$ for some $x, y \in S$. Hence we obtain that

$$
a=a b^{\prime} a=x b \cdot b^{\prime} \cdot b y=x b y=x \cdot b y=x a .
$$

$(\mathrm{x}) \Rightarrow(\mathrm{xi})$ For $a^{\prime} \in V(a)$ we have $a=a a^{\prime} a=a a^{\prime} \cdot x b=e b$ with $e=a a^{\prime} x \in E_{S}$ since

$$
e^{2}=a a^{\prime} x \cdot a a^{\prime} x=a a^{\prime} \cdot x a \cdot a^{\prime} x=a a^{\prime} \cdot a \cdot a^{\prime} x=a a^{\prime} x=e .
$$

(xi) $\Rightarrow$ (i) For $a^{\prime} \in V(a)$ we have $a=a a^{\prime} a=b x \cdot a^{\prime} a=b f$ with $f=x a^{\prime} a \in E_{S}$ since because $a=e b$ we have $e a=a$ and thus

$$
f^{2}=x a^{\prime} a \cdot x a^{\prime} a=x a^{\prime} \cdot e b \cdot x a^{\prime} a=x a^{\prime} e \cdot a=x a^{\prime} \cdot e a=x a^{\prime} \cdot a=f .
$$

REMARK. One can also add the right-left dual conditions. 
2. The generalization. Using the equivalent properties of the natural partial order on regular semigroups it is now possible to define a natural partial order on any semigroup. Evidently, any one of the conditions in Lemma 1 allows a generalization to arbitrary semigroups $S$. The resulting relation will not be a partial order on $S$, in general. But with the exception of (vii)-(ix) all of them are still antisymmetric relations. We give a proof for the case of condition (i); all the other cases are similar.

Proposition 2. Let $(S, \cdot)$ be any semigroup. Then the relation $a \sigma b$ iff $a=$ $e b=b f$ for some $e, f \in E_{S^{1}}$ is reflexive and antisymmetric on $S$.

ProOF. Let $\left(T_{S^{1}}, \circ\right)$ be the semigroup of all mappings from $S^{1}$ into $S^{1}$ with respect to composition of functions, where $S^{1}$ denotes the set $S$ if $(S, \cdot)$ has an identity, and the set $S$ with an identity adjoined in the other case. Consider the extended right-regular representation of $S$ (see Howie [6]), i.e.

$\varphi: S \rightarrow T_{S^{1}}, \quad a \varphi=f_{a}$ for all $a \in S, \quad x f_{a}=x a$ for all $x \in S^{1}$.

Suppose now that $a \sigma b$ and $b \sigma a$ for some elements $a, b \in S$. Thus there are $e, f, g, h \in E_{S^{1}}$ such that $a=e b=b f$ and $b=g a=a h$. Since $\varphi$ is a homomorphism we get

$$
a \varphi=(e \varphi)(b \varphi)=(b \varphi)(f \varphi), \quad b \varphi=(g \varphi)(a \varphi)=(a \varphi)(h \varphi) .
$$

Since idempotents of $(S, \cdot)$ are mapped onto idempotents of $\left(T_{S^{1}}, 0\right)$ and since $\left(T_{S^{1}}, \circ\right)$ is a regular semigroup, we obtain by Lemma $1(\mathrm{i})$ that $a \varphi \preccurlyeq b \varphi$ and $b \varphi \preccurlyeq a \varphi$ in the natural partial order of $\left(T_{S^{1}}, \circ\right)$. Hence $a \varphi=b \varphi$ and $a=b$, because $\varphi$ is one-to-one.

Examples of semigroups for which $\sigma$ is even a partial order are semigroups $S$ such that $E_{S}$ forms a subsemigroup of $S$. In particular, for every commutative semigroup, $\sigma$ is a partial order which is compatible with multiplication, also. Using condition $(x)$ we get

THEOREM 3. For any semigroup $(S, \cdot)$ the relation " $\leq$ " defined by: $a \leq b$ iff $a=x b=b y, x a=a$ for some $x, y \in S^{1}$, is a partial order on $S$ called the natural partial order of $(S, \cdot)$.

PROOF. Antisymmetry is proved as in Proposition 2. For the proof of transitivity let $a, b \in S$ such that $a \leq b$ and $b \leq a$. Then there are $x, y, w, z \in S^{1}$ such that

$$
a=x b=b y, \quad x a=a \quad \text { and } \quad b=w c=c z, \quad w b=b .
$$

Hence, $a=(x w) c=c(z y)$ and

$$
(x w) a=(x w) b y=x \cdot w b \cdot y=x \cdot b \cdot y=x \cdot b y=x \cdot a=a
$$

thus $a \leq c$.

COROLlaRY. The natural partial order on the semigroup $(S, \cdot)$ coincides with relation (3) above if $(S, \cdot)$ is regular. It is equal to relation (1) above when restricted to the set $E_{S}$ of idempotents.

PROOF. The proof is by application of Lemma 1.

The natural partial order is not compatible with multiplication on an arbitrary semigroup since it is not so for regular semigroups. 
3. A characterization. The most obvious definition of a partial order on an arbitrary semigroup $(S, \cdot)$ arises by the regular representation of $S$ mentioned above. It is not a natural partial order in the strict sense. But it is tightly connected with the natural partial order. In order to define it, consider again the extended rightregular representation $\varphi$ of the semigroup $(S, \cdot)$ (see the proof of Proposition 2). Since $\left(T_{S^{1}}\right.$, o) is a regular semigroup, it is naturally partially ordered by any of the equivalent relations of Lemma 1 . This ordering $\preccurlyeq$ can be characterized in terms of mappings (Theorem 2.2 of Kowol and Mitsch [7]); namely, for $f, g \in T_{S^{1}}$,

$f \preccurlyeq g$ iff (i) $\operatorname{Im} f \subseteq \operatorname{Im} g$, (ii) $\operatorname{Ker} f \geq \operatorname{Ker} g$, (iii) if $x g \in \operatorname{Im} f$ for some $x \in S^{1}$ then $x g=x f$.

Here $\operatorname{Im} f$ denotes the range of $f$ and $\operatorname{Ker} f$ the equivalence relation on $S^{1}$ associated with the mapping $f$, i.e. $x(\operatorname{Ker} f) y$ iff $x f=y f$. Now define a relation $\tau_{1}$ on $S$ by

$$
\begin{aligned}
a \tau_{1} b \quad \text { iff } & a \varphi \preccurlyeq b \varphi, \\
& \text { iff } f_{a} \preccurlyeq f_{b}, \\
& \text { iff } \\
& \text { (i) } S^{1} a \subseteq S^{1} b,\left(\text { ii) } x b=y b\left(x, y \in S^{1}\right) \Rightarrow x a=y a,\right. \\
& \text { (iii) } x b \in S^{1} a\left(x \in S^{1}\right) \Rightarrow x b=x a .
\end{aligned}
$$

Similarly, using the extended left-regular representation of the semigroup $(S, \cdot)$ by means of left translations $f^{a} \in T_{S^{1}}$ given by $x f^{a}=a x$ for all $x \in S^{1}$ and using the dual composition of functions on $T_{S^{1}}$, define a relation $\tau_{2}$ on $S$ by

$$
a \tau_{2} b \text { iff } f^{a} \preccurlyeq f^{b} .
$$

Evidently, the relations $\tau_{1}$ and $\tau_{2}$ are partial orders on any semigroup $(S, \cdot)$ since $\preccurlyeq$ is a partial order on $T_{S^{1}}$. It will be shown that the natural partial ordering on $(S, \cdot)$ is equal to the intersection of $\tau_{1}$ and $\tau_{2}$.

If $\rho$ denotes any of the relations defined by conditions (i)-(vi), (x) and (xi), respectively (Lemma 1 ), then $\rho$ is finer than $\tau_{1}$ and $\tau_{2}$ in the usual ordering of binary relations. In fact, let $a \rho b$ for some $a, b \in S$. Applying the homomorphism $\varphi: S \rightarrow T_{S^{1}}$ to any of the defining equations of $\rho$ one obtains an equation in $\left(T_{S^{1}}, \circ\right)$. By Lemma 1 they each imply that $a \varphi \preccurlyeq b \varphi$ in the natural partial order $\preccurlyeq$ of $\left(T_{S^{1}}, \circ\right)$. Hence $a \tau_{1} b$ and $a \tau_{2} b$, respectively. Consequently, in the lattice of all binary relations on $S, \rho \subseteq \tau_{1}$ and $\rho \subseteq \tau_{2}$ imply that $\rho \subseteq \tau_{1} \cap \tau_{2}$. In particular, the natural partial order $\leq$ on $S$ is finer than $\tau=\tau_{1} \cap \tau_{2}$. Conversely, let $a \tau b$ for some $a, b \in S$. Then $a \tau_{1} b$ and $a \tau_{2} b$, so that by (i) of (4) and (5) we obtain $a=x b=b y$ for some $x, y \in S^{1}$. Hence, $x b=a \in S^{1} a$ implies by (iii) of (4) that $a=x b=x a$ and $a \leq b$. Thus, $\leq$ coincides with $\tau=\tau_{1} \cap \tau_{2}$.

THEOREM 4. For any semigroup $(S, \cdot)$ the natural partial order is equal to the relation $\tau=\tau_{1} \cap \tau_{2}$ defined by (4) and (5) above.

COROLLARY. For any semigroup $(S, \cdot)$ and its natural partial order the following conditions are equivalent:

(i) $a \leq b$,

(ii) $a=w b=b z, a z=a$ for some $w, z \in S^{1}$,

(iii) $a=x b=b y, x a=a y=a$ for some $x, y \in S^{1}$. 

(5).

PROOF. The proof is by the last argument preceding Theorem 4 using (4) and

REMARKS. (1) If the semigroup $(S, \cdot)$ satisfies the right (left) cancellation law, then its natural partial order is trivial, but $\tau_{1}\left(\tau_{2}\right)$ is compatible on the right (left) with multiplication. Hence, $S$ is a right (left) partially ordered semigroup with respect to $\tau_{1}\left(\tau_{2}\right)$.

(2) László Fuchs gave the following direct proof of the Corollary to Theorem 4:

$$
a \leq b \Rightarrow a=x a=b y, \quad x a=a \Rightarrow a y=x b \cdot y=x \cdot b y=x \cdot a=a .
$$

He pointed out also that by use of property (iii) of the natural partial order on any semigroup $(S, \cdot)$ (Corollary to Theorem 4 ) the antisymmetry of $\leq$ can be shown as follows:

$a \leq b$ and $b \leq a$

$$
\begin{aligned}
& \Rightarrow a=x b=b y, x a=a y=a \text { and } b=w a=a z, w b=b z=b \\
& \Rightarrow b=w a=w \cdot a y=w a \cdot y=b \cdot y=a .
\end{aligned}
$$

(3) If the semigroup $(S, \cdot)$ is periodic (in particular, finite) then the natural partial order on $S$ is given by

$$
a \leq b \quad \text { iff } \quad a=e b=b f \quad \text { for some } e, f \in E_{S^{1}}
$$

and coincides with the natural partial order (3) of regular semigroups.

\section{REFERENCES}

1. T. Blyth and G. Gomes, On the compatibility of the natural partial order on a regular semigroup, Proc. Roy. Soc. Edinburgh 94 (1983), 79-84.

2. W. Burgess and R. Raphael, On Conrad's partial order relation on semiprime rings and on semigroups, Semigroup Forum 16 1978, 133-140.

3. G. Gomes, On left quasinormal orthodox semigroups, Proc. Roy. Soc. Edinburgh 95 (1983), 59-71.

4. R. Hartwig, How to partially order regular elements, Math. Japon. 25 (1980), 1-13.

5. J. Hickey, Semigroups under a sandwich operation, Proc. Edinburgh Math. Soc. 26 (1983), 371-382.

6. J. Howie, An introduction to semigroup theory, Academic Press, New York, 1976.

7. G. Kowol and H. Mitsch, Naturally ordered full transformation semigroups, Monatsh. Math. (to appear).

8. M. Lawson, The natural partial order on an abundant semigroup, preprint, Univ. of York, England, 1984.

9. D. McAlister, Regular Rees matrix semigroups and regular Dubreil-Jacotin semigroups, J. Austral. Math. Soc. 31 (1981), 325-336.

10. K. Nambooripad, The natural partial order on a regular semigroup, Proc. Edinburgh Math. Soc. 23 (1980), 249-260.

11. V. Vagner, Generalized groups, Dokl. Akad. Nauk SSSR 84 (1952), 1119-1122. (Russian)

Department of MAThematics, University of Vienna, 1090 Wien, AUstria 